\title{
Publisher's Note: “Electric field controlled Faraday rotation in an electro-optic/magneto-optic bilayer" [Appl. Phys. Lett. 97, 011901 (2010)]
}

\author{
Yu. S. Dadoenkova, , ,a) I. L. Lyubchanskii, ${ }^{1, b)}$ Y. P. Lee, ${ }^{2, c)}$ and Th. Rasing ${ }^{3, d)}$ \\ ${ }^{1}$ Donetsk Physical and Technical Institute of the NAS of Ukraine, 83114 Donetsk, Ukraine and Department \\ of Physics, Donetsk National University, 83000 Donetsk, Ukraine \\ ${ }^{2}$ Department of Physics, Quantum Photonic Science Research Center (q-Psi), Hanyang University, \\ Seoul 133-791, Republic of Korea \\ ${ }^{3}$ Institute for Molecules and Materials, Radboud University Nijmegen, 6525 AJ Nijmegen, The Netherlands
}

(Received 16 August 2010; published online 16 September 2010)

[doi:10.1063/1.3488679]

This article was originally published online and in print with an incomplete sentence in the abstract and a typographical error in the acknowledgment section. In the abstract, the first sentence should have read, "The Faraday rotation for light transmission through a bilayered structure composed of an electro-optic semiconductor film and a slab with high values of magneto-optic and magnetoelectric interactions is theoretically investigated." instead of "The Faraday rotation for light transmission through a bilayered structure composed of an electro-optic semiconductor film and a slab with high values of magneto-optic and magnetoelectric interactions;" in the acknowledgment section, "NWO" appeared incorrectly as "NOW." AIP apologizes for these errors. All online versions of the article have been corrected.

\footnotetext{
${ }^{a)}$ Electronic mail: yulidad@fti.dn.ua.

${ }^{b)}$ Electronic mail: igorl@fti.dn.ua.

${ }^{c)}$ Electronic mail: yplee@ hanyang.ac.kr.

${ }^{\mathrm{d})}$ Electronic mail: th.rasing@ @ science.ru.nl.
} 